\title{
Light curing through glass ceramics with a second- and a third- generation LED curing unit: effect of curing mode on the degree of conversion of dual-curing resin cements
}

\author{
Simon Flury • Adrian Lussi • Reinhard Hickel • \\ Nicoleta Ilie
}

Received: 23 July 2012 / Accepted: 21 January 2013 / Published online: 8 February 2013

(C) Springer-Verlag Berlin Heidelberg 2013

\begin{abstract}
Objectives The aim of this study was to measure the degree of conversion (DC) of five dual-curing resin cements after different curing modes with a second- and a third-generation light-emitting diode (LED) curing unit. Additionally, irradiance of both light curing units was measured at increasing distances and through discs of two glass ceramics for computer-aided design/manufacturing (CAD/CAM).

Materials and methods Irradiance and spectra of the Elipar FreeLight 2 (Standard Mode (SM)) and of the VALO light curing unit (High Power Mode (HPM) and Xtra Power Mode (XPM)) were measured with a MARC radiometer. Irradiance was measured at increasing distances (control) and through discs (1.5 to $6 \mathrm{~mm}$ thickness) of IPS Empress CAD and IPS e.max CAD. DC of Panavia F2.0, RelyX Unicem 2 Automix, SpeedCEM, BisCem, and BeautiCem SA was measured with an attenuated total reflectance-

\author{
S. Flury $(\bowtie) \cdot$ A. Lussi \\ Department of Preventive, Restorative and Pediatric Dentistry, \\ School of Dental Medicine, University of Bern, Freiburgstrasse 7, \\ 3010, Bern, Switzerland \\ e-mail: simon.flury@zmk.unibe.ch \\ A. Lussi \\ e-mail: adrian.lussi@zmk.unibe.ch \\ R. Hickel $\cdot$ N. Ilie \\ Department of Restorative Dentistry, Dental School of the \\ Ludwig-Maximilians-University, Goethestrasse 70, \\ R. Hickel \\ e-mail: hickel@dent.med.uni-muenchen.de \\ N. Ilie \\ e-mail: nicoleta.ilie@dent.med.uni-muenchen.de
}

80336, Munich, Germany
\end{abstract}

Fourier transform infrared spectrometer when self-cured (negative control) or light cured in SM for $40 \mathrm{~s}$, HPM for $32 \mathrm{~s}$, or XPM for $18 \mathrm{~s}$. Light curing was performed directly (positive control) or through discs of either 1.5- or 3-mm thickness of IPS Empress CAD or IPS e.max CAD. DC was analysed with Kruskal-Wallis tests followed by pairwise Wilcoxon rank sum tests $(\alpha=0.05)$.

Results Maximum irradiances were $1,545 \mathrm{~mW} / \mathrm{cm}^{2}(\mathrm{SM})$, $2,179 \mathrm{~mW} / \mathrm{cm}^{2}$ (HPM), and 4,156 $\mathrm{mW} / \mathrm{cm}^{2}$ (XPM), and all irradiances decreased by $>80 \%$ through discs of $1.5 \mathrm{~mm}, \geq 95 \%$ through $3 \mathrm{~mm}$, and up to $>99 \%$ through $6 \mathrm{~mm}$. Generally, self-curing resulted in the lowest DC. For some cements, direct light curing did not result in higher DC compared to when light cured through ceramic discs. For other cements, light curing through ceramic discs of $3 \mathrm{~mm}$ generally reduced DC.

Conclusions Light curing was favourable for dual-curing cements. Some cements were more susceptible to variations in curing mode than others.

Clinical relevance When light curing a given cement, the higher irradiances of the third-generation LED curing unit resulted in similar DC compared to the second-generation one, though at shorter light curing times.

Keywords Luting resins · Polymerization · Light intensity · Curing light $\cdot$ Radiant exposure $\cdot$ Degree of cure

\section{Introduction}

Generally, glass ceramic materials such as leucite-reinforced or lithium-disilicate glass ceramics result in tooth restorations with good clinical success [1-3]. Moreover, computer- 
aided design/manufacturing (CAD/CAM) systems enable a simplified (i.e. direct, chairside) fabrication of glass ceramic restorations. However, challenges remain when working with glass ceramics as they need to be adhesively cemented to the remaining tooth structure by use of resin cements. These resin cements can basically be divided in three categories: (1) etch-and-rinse adhesive resin cements (cements used after application of an etch-and-rinse adhesive system including separate acid etching), (2) self-etch adhesive resin cements (cements used after application of a self-etch adhesive system), and (3) self-adhesive resin cements ("selfadhering" cements used without application of any adhesive system) [4]. Etch-and-rinse as well as self-etch adhesive resin cements are well documented in literature and may be regarded as gold standards. However, the necessity for application of an adhesive system implies numerous pre-treatment steps of the tooth structure. These pretreatment steps are technique sensitive and susceptible to handling errors. An error during the pre-treatment or during the application of the resin cements may lead to extensive or total failure of ceramic tooth restorations [4-6]. In order to simplify adhesive cementation, a third category of resin cements has been marketed, the selfadhesive resin cements, which require no pre-treatment steps at all.

Some etch-and-rinse and self-etch adhesive resin cements and most self-adhesive resin cements offer a so-called dual-curing ability. This allows curing initiated by a light source (light curing) as well as an automatic curing (self-curing), initiated by the mixing of two pastes. Previous studies have shown that light curing of dual-curing resin cements generally leads to improved physico-chemical properties compared to self-curing alone, i.e. to better micromechanical properties (a higher surface hardness or elastic modulus) [7,8], or to a higher degree of conversion [9]. Improved physico-chemical properties are clinically related to e.g. a decrease in marginal wear or discoloration $[10,11]$ as well as an increase in biocompatibility [12, 13]. However, when light curing through ceramic materials, the irradiance (also called "light intensity" or "power density") of the light curing unit decreases with increasing thickness of the ceramic material [14-16]. Thus, the actual irradiance reaching the dual-curing resin cement underneath the ceramic material is reduced to various extents, depending on the irradiance of the light curing unit and the thickness as well as the type and opacity of the ceramic material. A decrease in irradiance might deteriorate the physico-chemical properties of dual-curing resin cements due to a reduced effect of light curing which might not be compensated by their (generally poorer) self-curing ability. However, not only the irradiance reaching the resin cements but also the light curing time is important for curing. The product of irradiance $\left(\mathrm{mW} / \mathrm{cm}^{2}\right)$ and light curing time ( $\mathrm{s}$ ) is called radiant exposure (also called "energy density" or "energy dose"; J/ $\mathrm{cm}^{2}$ ). The radiant exposure consequently describes a simple reciprocal relationship: if irradiance is decreased, the light curing time must be increased [17-20]. Clinically, there is a demand for shorter light curing times, and thus, manufacturers have continuously strived for increased irradiances of their light curing units [21]. Today, a majority of light curing units is based on the light-emitting diode (LED) technology with a single high-powered diode (secondgeneration LED curing units; blue diode, single peak). Second-generation LED curing units typically reach irradiances of 1,200 to $1,500 \mathrm{~mW} / \mathrm{cm}^{2}$. The third-generation of LED curing units contains multiple diodes (violet/blue diodes, polywave), and recently a third-generation LED curing unit was marketed (VALO, Ultradent), which is claimed to reach irradiances of up to $3,200 \mathrm{~mW} / \mathrm{cm}^{2}$ depending on the chosen mode.

Literature is rather sparse as regards whether/to what extent (a) the degree of conversion (DC) of dual-curing resin cements is influenced by shorter light curing times with higher irradiances of a third-generation compared to a second-generation LED curing unit under near identical radiant exposures, and to what extent (b) the higher irradiances of a third-generation compared to a secondgeneration LED curing unit is transmitted through CAD/CAM glass ceramic materials of increasing thickness. Therefore, the primary aim of this study was to measure and statistically analyse DC of one dual-curing, self-etch adhesive resin cement and four dual-curing, self-adhesive resin cements when cured according to 16 curing modes comprising self-curing (negative control), direct light curing (positive control), and light curing through discs of two different thicknesses of a leucite-reinforced and a lithium-disilicate glass ceramic material for CAD/CAM. Light curing was chosen to be performed with the second-generation LED curing unit Elipar FreeLight 2 and the third-generation LED curing unit VALO under near identical radiant exposures. For calculation of radiant exposures and as a secondary aim, irradiances of both light curing units were measured at increasing distances without any ceramic disc between the tip end of the light curing unit and the radiometer (i.e. through air, as control) as well as through discs of increasing thickness of the two glass ceramic materials, and the irradiances were descriptively analysed. Furthermore, spectra of the second-generation LED curing unit Elipar FreeLight 2 (blue) and the third-generation LED curing unit VALO (violet/blue) were measured for documentation.

The null hypothesis for measurement of DC was that all five resin cements would yield an equal DC regardless of the curing mode. 


\section{Materials and methods}

Preparation of ceramic discs

One block of a leucite-reinforced glass ceramic material (IPS Empress CAD, size C14, shade LT A3; Ivoclar Vivadent, Schaan, Liechtenstein) and one block of a lithiumdisilicate glass ceramic material (IPS e.max CAD, size C14, shade LT A3; Ivoclar Vivadent) was used for the ceramic discs. The block of the IPS e.max CAD ceramic material was tempered in a Programat EP 5000 furnace (Ivoclar Vivadent) using the standard program recommended by the manufacturer (G9 Crystal/Glaze, program P81). From the two blocks of ceramic material, discs were cut using a low-speed diamond saw (IsoMet, Lake Bluff, IL, USA). The ceramic discs were then polished under water cooling on both sides with a polishing device (Leco VP 100; Leco Instrumente $\mathrm{GmbH}$, Mönchengladbach, Germany) and silicon carbide papers (Leco abrasive discs; Leco) of descending grit size (\#320 to \#1200). During polishing, the thickness of the ceramic discs was monitored with a digital micrometer (Mitutoyo ID-U1025; Mitutoyo, Kawasaki, Japan). The discs were polished until the following thicknesses were reached (one ceramic disc per thickness of each of the two ceramic materials): $1.5,3.0,4.5$, and $6.0 \mathrm{~mm}$.

\section{Measurement of irradiance and spectra}

Irradiance and spectra were measured of the Elipar FreeLight 2 light curing unit (3M ESPE, Seefeld, Germany) in the Standard Mode and the VALO light curing unit (Ultradent, South Jordan, UT, USA) in the High Power Mode as well as in the Xtra Power Mode. Irradiance of the three modes was measured either without the ceramic discs (i.e. through air, as a control) at a distance of $0 \mathrm{~mm}$ and (using space holders) at increasing distances of 1, 2, 3, 4, 5, and $6 \mathrm{~mm}$ or with the ceramic discs of $1.5,3,4.5$, and $6 \mathrm{~mm}$ thickness inserted between the light curing unit and the radiometer device. Five measurements per distance were made using a radiometer device (MARC Resin Calibrator, BlueLight Analytics Inc., Halifax, NS, Canada).

Spectra of the three modes were measured without the ceramic discs at a distance of $0 \mathrm{~mm}$ with the same radiometer device.

\section{Calculation of radiant exposure}

For the Elipar FreeLight 2 light curing unit, a clinically relevant light curing time of $40 \mathrm{~s}$ (in two subsequent cycles of $20 \mathrm{~s}$ each) was chosen. Measurements of irradiance of the Elipar FreeLight 2 light curing unit at a distance of $0 \mathrm{~mm}$ showed a mean irradiance of $1,545 \mathrm{~mW} / \mathrm{cm}^{2}$. Consequently, the radiant exposure (i.e. irradiance $\times$ light curing time; $\mathrm{J} / \mathrm{cm}^{2}$ ) for the Elipar FreeLight 2 light curing unit was $61.8 \mathrm{~J} / \mathrm{cm}^{2}$. For the VALO light curing unit, the High Power Mode at a distance of $0 \mathrm{~mm}$ showed a mean irradiance of $1,869 \mathrm{~mW} / \mathrm{cm}^{2}$, and the Xtra Power Mode showed a mean irradiance of $3,505 \mathrm{~mW} / \mathrm{cm}^{2}$. In order to have near identical radiant exposures with the three light curing units/modes, a light curing time of $32 \mathrm{~s}$ (in eight subsequent cycles of $4 \mathrm{~s}$ each; $59.8 \mathrm{~J} / \mathrm{cm}^{2}$ ) was set for the High Power Mode, and a light curing time of $18 \mathrm{~s}$ (in six subsequent cycles of $3 \mathrm{~s}$ each; $63.1 \mathrm{~J} / \mathrm{cm}^{2}$ ) was set for the Xtra Power Mode of the VALO light curing unit.

Resin cements and curing modes

One dual-curing, self-etch adhesive resin cement (control) and four dual-curing, self-adhesive resin cements were used. Detailed information about the resin cements is listed in Table 1. The self-etch adhesive resin cement (Panavia F2.0) was hand mixed according to the manufacturer's instruction with a $1: 1$ ratio of paste $A$ and paste $B$. The four self-adhesive resin cements (in Automix syringes) were used with the mixing tips delivered by the manufacturers, and the first $\sim 1 \mathrm{~cm}$ of resin cement of each new Automix syringe was discarded. Resin cements which needed to be stored in the refrigerator were taken out $1 \mathrm{~h}$ before measurement of DC.

The five resin cements were cured according to 16 groups of curing mode listed in Table 2. Self-curing of the self-etch adhesive resin cement Panavia F2.0 was either performed with addition of ED Primer II (group SC*) or without (group SC; Tables 1 and 2). When self-curing of Panavia F2.0 was performed with ED Primer II, liquid A and liquid $\mathrm{B}$ were mixed according to the manufacturer's instruction with a 1:1 ratio. A droplet of mixed ED Primer II was added to paste A and B of Panavia F2.0 with a probe and hand mixed. Light curing was performed either directly through a Mylar strip (positive control) or through the ceramic discs of 1.5- or 3-mm thickness. These two thicknesses were considered to be clinically most relevant.

\section{Measurement of DC}

To determine the DC, a plastic mould of $200 \mu \mathrm{m}$ thickness was placed in a Fourier transform infrared (FTIR) spectrometer with an attenuated total reflectance (ATR) accessory (Nexus, Thermo Nicolet, Madison, WI, USA). The mould was then filled with one of the five resin cements (Table 1) and covered with a Mylar strip. The top side of the resin cement was made flush with the mould by use of a small glass slide. This slide was removed, and the resin cement was cured according to one of the 16 groups of curing mode previously described (Table 2). Custom-made jigs had been made of 

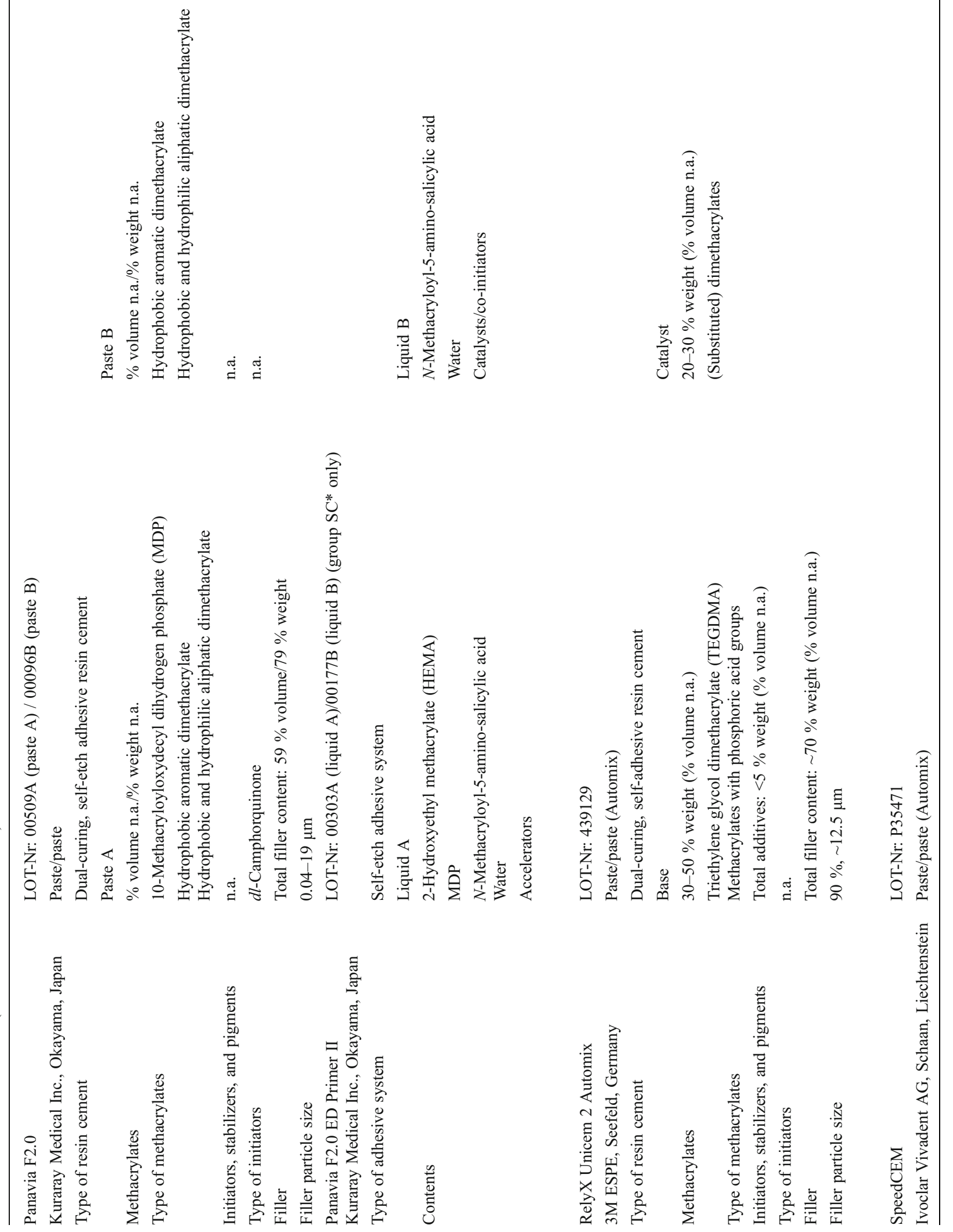


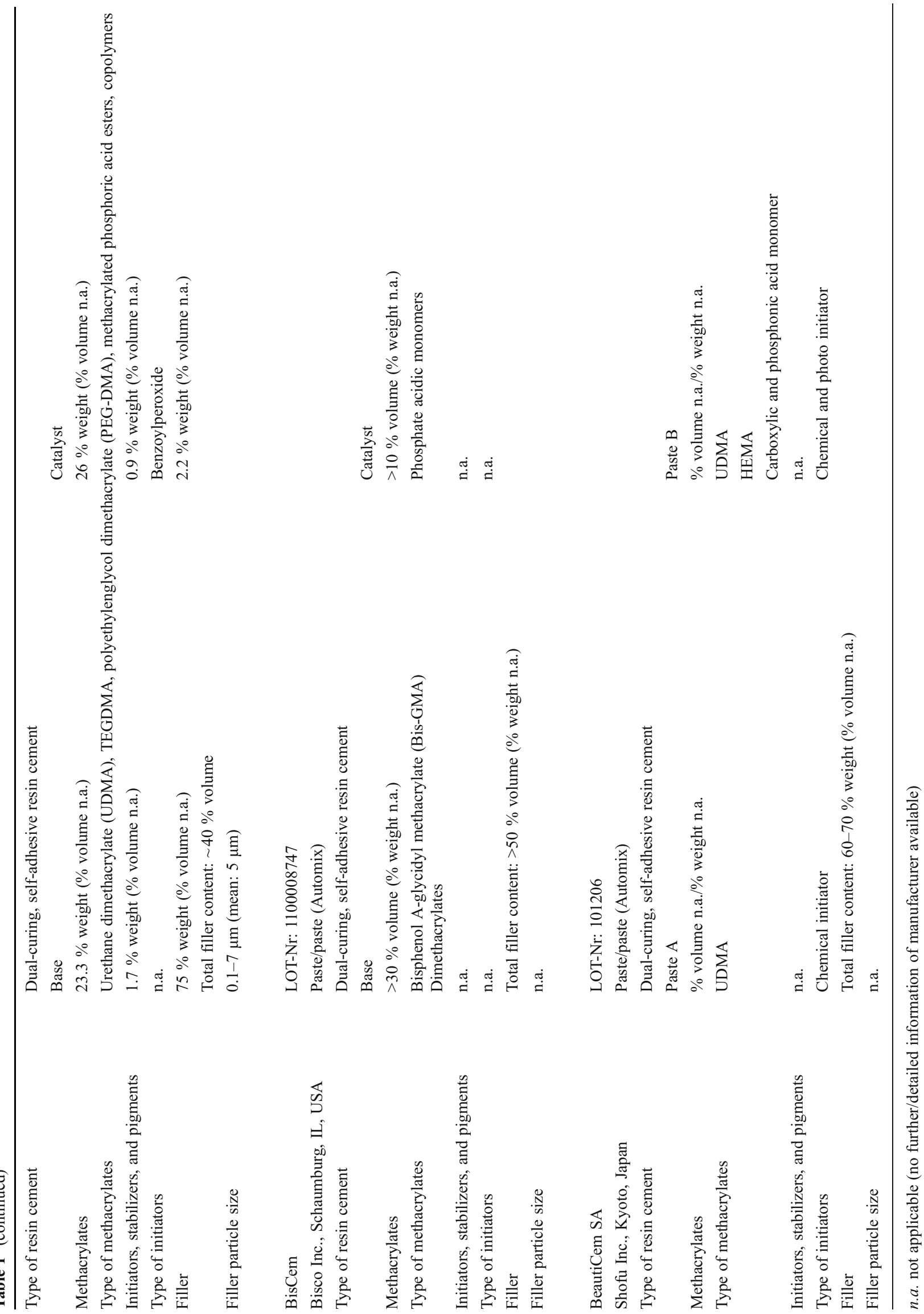


Table 2 Mean values and standard deviations of the degree of conversion (DC) of the five resin cements according to the groups of curing mode as well as results of the pairwise Wilcoxon rank sum tests (significance level $\alpha=0.05$; identical letters indicate no statistically significant differences between the groups within one resin cement)

\begin{tabular}{|c|c|c|c|c|c|c|}
\hline \multicolumn{2}{|l|}{ Resin cement } & \multirow{2}{*}{$\begin{array}{l}\text { Panavia F2.0 } \\
\text { DC (\%) }\end{array}$} & \multirow{2}{*}{$\begin{array}{l}\text { RelyX Unicem } \\
2 \text { Automix } \\
\text { DC }(\%)\end{array}$} & \multirow{2}{*}{$\begin{array}{l}\text { SpeedCEM } \\
\text { DC }(\%)\end{array}$} & \multirow{2}{*}{$\begin{array}{l}\text { BisCem } \\
\text { DC }(\%)\end{array}$} & \multirow{2}{*}{$\begin{array}{l}\text { BeautiCem SA } \\
\text { DC }(\%)\end{array}$} \\
\hline Light curing unit & $\begin{array}{l}\text { Groups of curing mode } \\
\text { ( } n=6 \text { per group and resin cement) }\end{array}$ & & & & & \\
\hline n.a.; self-curing & Group SC* (Panavia F2.0 only) & $60.1(4.3) \mathrm{a}$ & (n.a.) & (n.a.) & (n.a.) & (n.a.) \\
\hline n.a.; self-curing & Group SC & $29.4(9.1) \mathrm{e}$ & $24.7(2.8) \mathrm{j}$ & $56.4(7.0) \mathrm{c}$ & $18.9(9.7) \mathrm{f}$ & 54.5 (2.2) cdefg \\
\hline \multirow{5}{*}{$\begin{array}{l}\text { Elipar FreeLight } 2 \\
\text { (3M ESPE) Standard } \\
\text { Mode } 40 \mathrm{~s}(2 \times 20 \mathrm{~s})\end{array}$} & $\begin{array}{l}\text { Group EF1 } \\
\text { (direct; through Mylar strip) }\end{array}$ & 46.5 (2.9) bcd & $51.1(1.3) \mathrm{a}$ & $65.7(1.2) a b$ & $54.8(2.7)$ bcde & $57.3(2.8) \mathrm{ab}$ \\
\hline & $\begin{array}{l}\text { Group EF2 } \\
\text { (IPS Empress CAD } 1.5 \mathrm{~mm} \text { ) }\end{array}$ & $45.6(3.5) \mathrm{bcd}$ & $50.2(1.5)$ abd & $65.5(2.7) \mathrm{ab}$ & $54.8(1.7) \mathrm{bcd}$ & $55.1(1.0)$ bcdh \\
\hline & $\begin{array}{l}\text { Group EF3 } \\
\text { (IPS Empress CAD } 3 \mathrm{~mm} \text { ) }\end{array}$ & $42.3(2.8) \mathrm{d}$ & $47.9(1.1)$ efg & $65.1(2.1) \mathrm{ab}$ & $53.7(2.8)$ bcde & 52.3 (2.3) ghi \\
\hline & $\begin{array}{l}\text { Group EF4 } \\
\text { (IPS e.max CAD } 1.5 \mathrm{~mm} \text { ) }\end{array}$ & $45.5(3.0) \mathrm{bcd}$ & 49.5 (1.8) aef & $65.8(1.8) \mathrm{ab}$ & $56.4(2.6) \mathrm{ab}$ & $56.4(2.3) \mathrm{abc}$ \\
\hline & Group EF5 (IPS e.max CAD 3 mm) & $42.0(4.0) \mathrm{d}$ & $44.2(1.5) \mathrm{i}$ & $64.6(2.4) \mathrm{ab}$ & $54.8(2.6)$ bcde & 53.4 (2.4) dgi \\
\hline \multirow{5}{*}{$\begin{array}{l}\text { VALO (Ultradent) } \\
\text { High Power Mode } \\
32 \mathrm{~s}(8 \times 4 \mathrm{~s})\end{array}$} & $\begin{array}{l}\text { Group VH1 } \\
\text { (direct; through Mylar strip) }\end{array}$ & $48.7(2.4) b$ & $50.9(1.4) \mathrm{ab}$ & $63.4(2.6) \mathrm{b}$ & $58.4(1.2) \mathrm{a}$ & $58.2(2.4)$ af \\
\hline & $\begin{array}{l}\text { Group VH2 } \\
\text { (IPS Empress CAD } 1.5 \mathrm{~mm} \text { ) }\end{array}$ & $48.6(3.1) \mathrm{b}$ & $49.2(1.3)$ aef & $67.9(2.7)$ a & $56.4(2.4)$ abcd & $58.1(2.7)$ af \\
\hline & $\begin{array}{l}\text { Group VH3 } \\
\text { (IPS Empress CAD } 3 \mathrm{~mm} \text { ) }\end{array}$ & $46.8(2.7) \mathrm{bc}$ & 47.4 (2.2) cefgh & $64.1(2.7) \mathrm{b}$ & $55.3(2.7) \mathrm{bcd}$ & 55.4 (2.9) adefg \\
\hline & $\begin{array}{l}\text { Group VH4 } \\
\text { (IPS e.max CAD } 1.5 \mathrm{~mm} \text { ) }\end{array}$ & 47.7 (2.9) b & 50.5 (1.6) abc & $66.3(3.8) \mathrm{ab}$ & $56.4(3.7)$ abcd & $58.5(2.5) \mathrm{a}$ \\
\hline & $\begin{array}{l}\text { Group VH5 } \\
\text { (IPS e.max CAD } 3 \mathrm{~mm} \text { ) }\end{array}$ & $42.0(3.5) \mathrm{d}$ & $45.6(1.4) \mathrm{h}$ & $63.7(3.4) a b$ & $52.5(2.0) \mathrm{de}$ & $53.8(2.4) \mathrm{cg}$ \\
\hline \multirow{5}{*}{$\begin{array}{l}\text { VALO (Ultradent) } \\
\text { Xtra Power Mode } \\
18 \mathrm{~s}(6 \times 3 \mathrm{~s})\end{array}$} & $\begin{array}{l}\text { Group VX1 } \\
\text { (direct; through Mylar strip) }\end{array}$ & $47.6(2.6) \mathrm{b}$ & 49.1 (1.0) bef & $64.2(1.0) \mathrm{ab}$ & $56.8(2.9) \mathrm{abc}$ & 57.8 (3.1) abd \\
\hline & $\begin{array}{l}\text { Group VX2 } \\
\text { (IPS Empress CAD } 1.5 \mathrm{~mm} \text { ) }\end{array}$ & $48.5(3.7) b$ & $48.7(0.9)$ def & $66.4(2.1) a b$ & $54.5(1.2) \mathrm{cde}$ & $56.0(2.3)$ acd \\
\hline & $\begin{array}{l}\text { Group VX3 ( } \\
\text { IPS Empress CAD } 3 \text { mm) }\end{array}$ & $47.0(2.3) \mathrm{b}$ & 46.5 (1.5) gh & $65.5(2.3) \mathrm{ab}$ & $54.3(2.8)$ bcde & 53.1 (1.9) hg \\
\hline & $\begin{array}{l}\text { Group VX4 } \\
\text { (IPS e.max CAD } 1.5 \mathrm{~mm} \text { ) }\end{array}$ & $47.0(2.7) b$ & $50.0(1.0) \mathrm{ac}$ & $65.4(2.5) \mathrm{ab}$ & $54.7(2.7)$ bcde & $55.2(2.0)$ abcdefg \\
\hline & $\begin{array}{l}\text { Group VX5 } \\
\text { (IPS e.max CAD } 3 \mathrm{~mm} \text { ) }\end{array}$ & $43.4(2.9) \mathrm{cd}$ & $47.8(1.0)$ efg & $63.9(3.2) \mathrm{ab}$ & $52.2(1.8) \mathrm{e}$ & $50.7(1.9) \mathrm{i}$ \\
\hline
\end{tabular}

n.a. not applicable

acrylic resin (Paladur, pink shade; Heraeus Kulzer, Hanau, Germany) in order to ensure that the ceramic discs and the tip end of the light curing units were placed in the same position in the FTIR measuring area for all specimens. Simultaneously to curing, real-time measurements were carried out with the FTIR spectrometer, and FTIR spectra were recorded for $20 \mathrm{~min}$ (when self-cured; groups $\mathrm{SC}^{*}$ and $\mathrm{SC}$ ) and $5 \mathrm{~min}$ (when light cured; groups $\mathrm{EF}, \mathrm{VH}$, and $\mathrm{VX}$ ). For each of the five resin cements and 16 groups, six specimens were measured. The measuring area of the FTIR spectrometer was $800 \mu \mathrm{m}$ in diameter, the wave number of the FTIR spectra ranged from 4,000 to $650 \mathrm{~cm}^{-1}$, and the FTIR spectra were recorded with 2.36 spectra per second and two scans per spectrum at a resolution of $4 \mathrm{~cm}^{-1}$ (OMNIC Software, version 6.2; Thermo Nicolet). Measurement of DC was conducted at constant room temperature $\left(20{ }^{\circ} \mathrm{C}\right)$.
For Panavia F2.0, the variation in peak height ratio of the absorbance intensity of methacrylate carbon double bond peaks (at 1,637 $\mathrm{cm}^{-1}$ ) and of the absorbance intensity of aromatic carbon double bond peaks (at 1,608 $\mathrm{cm}^{-1}$ ) was assessed, and DC was calculated as follows:

$\mathrm{DC}(\%)=\left[\frac{\left(1,637 \mathrm{~cm}^{-1} / 1,608 \mathrm{~cm}^{-1}\right)_{\text {Peak height after curing }}}{\left(1,637 \mathrm{~cm}^{-1} / 1,608 \mathrm{~cm}^{-1}\right)_{\text {Peak height before curing }}}\right] \times 100$

For the other four resin cements, no aromatic carbon double bond peaks could be assessed. Thus, the variation in peak height ratio of methacrylate carbon double bond peaks of the recorded FTIR spectra before and after curing was assessed for calculation of DC.

During self-curing and after light curing had been performed, the measuring area of the FTIR spectrometer including the resin cement specimen was covered with a black 
photo-resistant box to avoid any additional curing effect of ambient light while measurement of DC continued.

\section{Statistical analysis of DC}

DC of all resin cements and curing modes were analysed using a nonparametrical ANOVA model with four fixed factors according to Brunner and Munzel [22]. The four fixed factors were: (1) resin cement (i.e. the five resin cements), (2) curing mode (i.e. self-curing, Elipar FreeLight 2 Standard Mode, VALO High Power Mode, and VALO Xtra Power Mode), (3) type of ceramic (i.e. no ceramic, IPS Empress CAD, IPS e.max CAD), and (4) ceramic thickness (i.e. no ceramic, $1.5 \mathrm{~mm}$ thickness, $3 \mathrm{~mm}$ thickness). The nonparametrical ANOVA model was followed by Kruskal-Wallis tests and pairwise Wilcoxon rank sum tests (significance level $\alpha=0.05$ ). No correction for multiple testing was done and thus, results must be considered exploratively. The entire statistical analysis was performed with SAS 9.2 (SAS Institute Inc., Cary, NC, USA).

\section{Results}

\section{Measurement of irradiance and spectra}

For the Elipar FreeLight 2 light curing unit in the Standard Mode (Fig. 1) without any ceramic disc, irradiance was $1,545 \mathrm{~mW} / \mathrm{cm}^{2}$ at $0-\mathrm{mm}$ distance, and it gradually decreased to $599 \mathrm{~mW} / \mathrm{cm}^{2}$ at $6-\mathrm{mm}$ distance. With the ceramic discs, the irradiance decreased through the $1.5-\mathrm{mm}$ discs (for IPS Empress CAD/IPS e.max CAD) by $83.3 \% / 86.5 \%$, through the 3 -mm discs by $95.5 \% / 96.8 \%$, through the 4.5 -mm discs by $99 \% / 99.4 \%$, and through the $6-\mathrm{mm}$ discs by $99.5 \% / 99.7 \%$. For the VALO light curing unit in the High

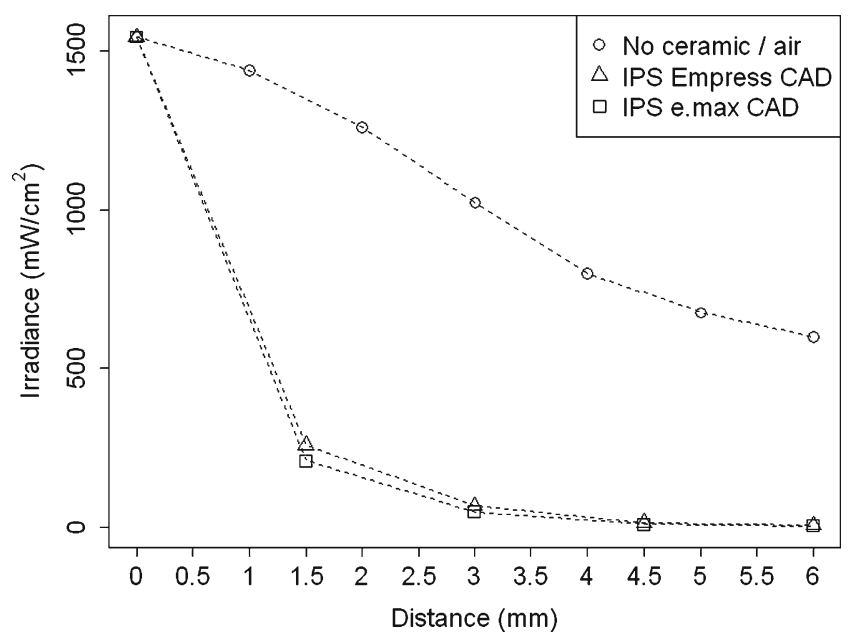

Fig. 1 Irradiance at increasing distances without ceramic discs as well as with the discs of the two ceramic materials for the Elipar FreeLight 2 light curing unit in the Standard Mode
Power Mode (Fig. 2) without any ceramic disc, irradiance was $1,869 \mathrm{~mW} / \mathrm{cm}^{2}$ at $0-\mathrm{mm}$ distance. Irradiance at 1 - and 2-mm distance was higher than at $0 \mathrm{~mm}$, and then it gradually decreased to $1,066 \mathrm{~mW} / \mathrm{cm}^{2}$ at $6-\mathrm{mm}$ distance. With the ceramic discs, there was no increase in irradiance: Compared to the irradiance without any ceramic disc at $0-\mathrm{mm}$ distance, the irradiance decreased through the $1.5-\mathrm{mm}$ discs (for IPS Empress CAD/IPS e.max CAD) by $82.3 \% / 85.8 \%$, through the 3 -mm discs by $95 \% / 96.4 \%$, through the 4.5 $\mathrm{mm}$ discs by $98.9 \% / 99.3 \%$, and through the $6-\mathrm{mm}$ discs by $99.5 \% / 99.7 \%$. Finally, for the VALO light curing unit in the Xtra Power Mode (Fig. 3) without any ceramic disc, irradiance was $3,505 \mathrm{~mW} / \mathrm{cm}^{2}$ at $0-\mathrm{mm}$ distance. Irradiance at 1- and 2-mm distance was higher than at $0 \mathrm{~mm}$, and then it gradually decreased to $2,072 \mathrm{~mW} / \mathrm{cm}^{2}$ at $6-\mathrm{mm}$ distance. Compared to the irradiance without any ceramic disc at 0 $\mathrm{mm}$ distance, the irradiance decreased through the $1.5-\mathrm{mm}$ discs (for IPS Empress CAD/IPS e.max CAD) by $81.9 \% / 85.8 \%$, through the 3 -mm discs by $95 \% / 96.5 \%$, through the $4.5-\mathrm{mm}$ discs by $98.9 \% / 99.3 \%$, and through the $6-\mathrm{mm}$ discs by $99.5 \% / 99.7 \%$.

Spectra of both light curing units and the three modes are shown in Fig. 4. The second-generation LED curing unit Elipar FreeLight 2 showed one distinct peak in the wavelength range of 440 to $460 \mathrm{~nm}$. In both modes, the thirdgeneration LED curing unit VALO showed a distinct smaller peak around $400 \mathrm{~nm}$ and a slightly stretched higher peak in the wavelength range of 440 to $470 \mathrm{~nm}$.

\section{Measurement of DC}

The nonparametrical ANOVA model with four fixed factors showed that all factors had a statistically significant effect on DC $(p<0.0001)$. Kruskal-Wallis tests showed significant

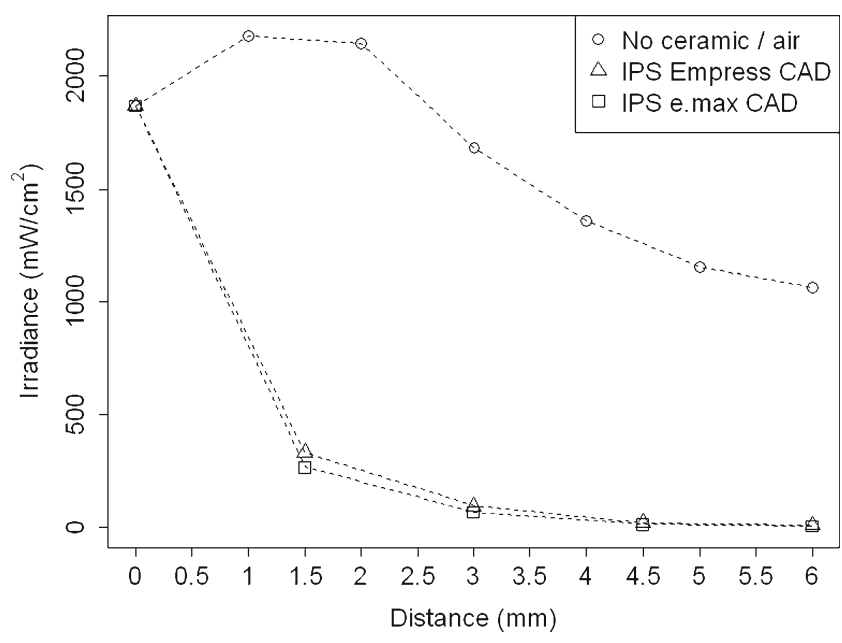

Fig. 2 Irradiance at increasing distances without ceramic discs as well as with the discs of the two ceramic materials for the VALO light curing unit in the High Power Mode 


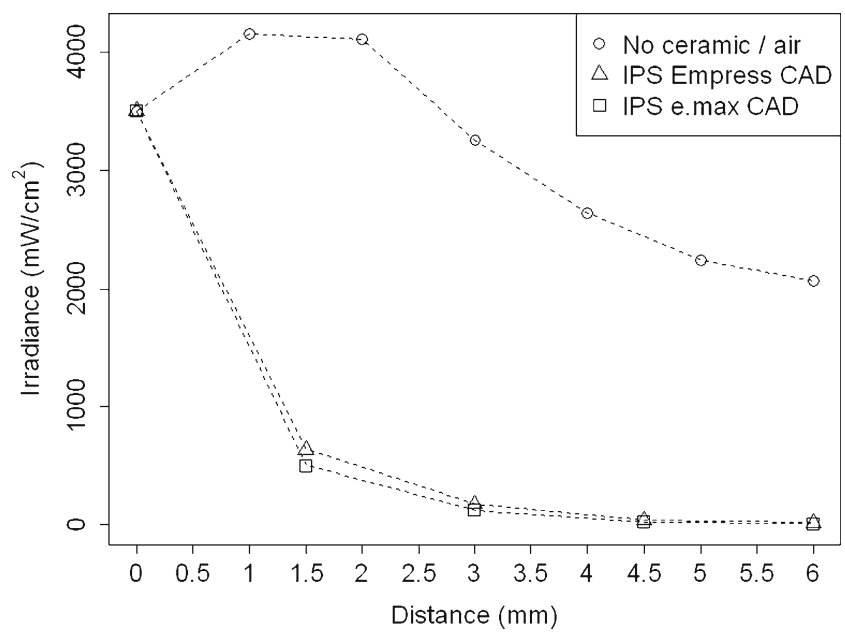

Fig. 3 Irradiance at increasing distances without ceramic discs as well as with the discs of the two ceramic materials for the VALO light curing unit in the Xtra Power Mode

differences between the resin cements as well as significant differences between curing modes within one resin cement. DC (mean values and standard deviations) as well as the results of the post hoc pairwise Wilcoxon rank sum tests are shown in Table 2 for all resin cements and groups of curing mode.

When comparing DC of the five resin cements, SpeedCEM resulted in the highest $\mathrm{DC}$, followed by BisCem and BeautiCem SA, and then by RelyX Unicem 2 Automix and Panavia F2.0. Within one resin cement, self-curing (group SC) led to significantly lower DC than did any of the curing modes involving light curing (groups EF, VH, and VX), with the exception of BeautiCem SA. For Panavia F2.0, self-curing with ED Primer II (group $\mathrm{SC}^{*}$ ) resulted in a significantly higher DC not only compared to self-curing without ED Primer II (group SC) but also compared to all

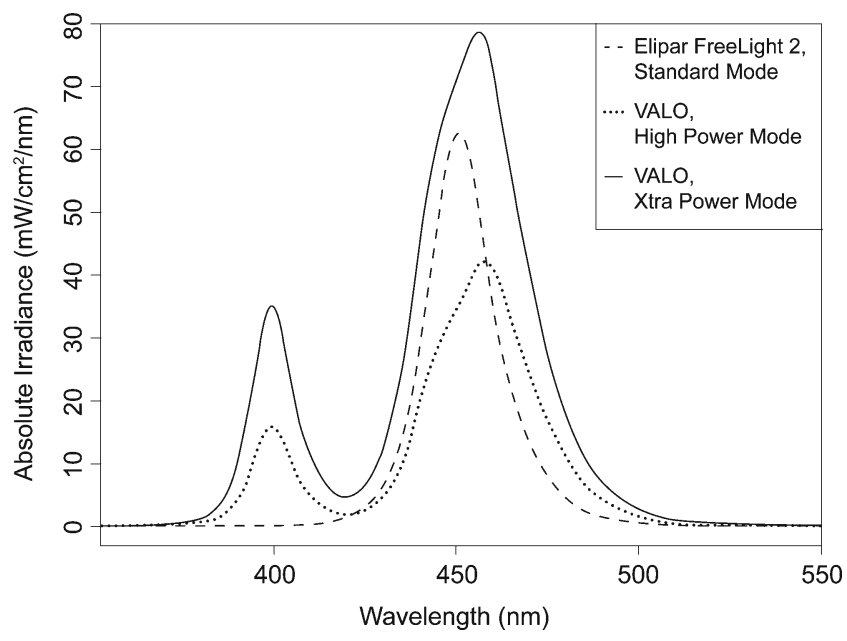

Fig. 4 Spectra without ceramic discs at a distance of $0 \mathrm{~mm}$ for the Elipar FreeLight 2 light curing unit in the Standard Mode, the VALO light curing unit in the High Power Mode, and the VALO light curing unit in the Xtra Power Mode groups involving light curing of Panavia F2.0. Generally, direct light curing through a Mylar strip (groups EF1, VH1, and VX1) did not lead to significantly higher DC compared to groups where light curing had been performed through a ceramic disc. Within one resin cement, Panavia F2.0, SpeedCEM, and BisCem generally showed no or only few significant differences in DC between the curing modes. For the other two resin cements (RelyX Unicem 2 Automix and BeautiCem SA), generally light curing through a $1.5-\mathrm{mm}$ ceramic disc did not lead to a significant decrease in DC, but light curing through a 3-mm ceramic disc resulted in significantly lower DC. Finally, there were few differences between the two ceramic materials as to their effect on DC.

\section{Discussion}

Measurement of irradiance was performed with a laboratory-grade radiometer, which is referenced by the National Institute of Standards and Technologies. When measured with this radiometer at $0-\mathrm{mm}$ distance, the Elipar FreeLight 2 in the Standard Mode reached a higher irradiance $\left(1,545 \mathrm{~mW} / \mathrm{cm}^{2}\right)$ than the irradiance of $1,200 \mathrm{~mW} / \mathrm{cm}^{2}$ stated by the manufacturer. This also applied to the VALO light curing unit $\left(1,869 \mathrm{~mW} / \mathrm{cm}^{2}\right.$ in the High Power Mode; stated by the manufacturer $1,400 \mathrm{~mW} / \mathrm{cm}^{2}$; and $3,505 \mathrm{~mW} / \mathrm{cm}^{2}$ in the Xtra Power Mode; stated by the manufacturer $3,200 \mathrm{~mW} / \mathrm{cm}^{2}$ ). Irradiance (i.e. as power $(\mathrm{mW})$ divided by area $\left(\mathrm{cm}^{2}\right)$ ) may vary depending on how it is measured. Beam profiles of light curing units are often inhomogeneous, the power being unequally distributed across the tip end. Thus, there are areas (centrically or circumferentially located) of higher power ("hot spots") and areas of lower power. If the power across the entire tip end is divided by the entire tip end area, the average irradiance will be lower than if the power of a hot spot is measured and divided by a smaller area. The sensor of the MARC radiometer has a diameter of $4 \mathrm{~mm}\left(\right.$ area $\left.=0.13 \mathrm{~cm}^{2}\right)$ whereas the tip end of the Elipar FreeLight 2 has a diameter of $8 \mathrm{~mm}$ (area $=0.5 \mathrm{~cm}^{2}$ ) and that of the VALO has a diameter of approximately $9 \mathrm{~mm}$ (area=0.64 $\mathrm{cm}^{2}$ ). The tip end of both light curing units was centrally placed on the sensor of the MARC radiometer. Thus, the higher irradiance measured in the present study compared to the irradiance stated by the manufacturers may be the result of a high centric power of the light curing units in combination with the smaller detection area of the MARC sensor.

In the present study, the VALO light curing unit in the Xtra Power Mode and without any ceramic disc reached a maximum irradiance of $4,156 \mathrm{~mW} / \mathrm{cm}^{2}$ at $1-\mathrm{mm}$ distance and an irradiance of $4,114 \mathrm{~mW} / \mathrm{cm}^{2}$ at $2-\mathrm{mm}$ distance. These findings are in accordance with the irradiance measured with a MARC radiometer in a study of Price et al. in which 
the VALO light curing unit reached $4,130 \mathrm{~mW} / \mathrm{cm}^{2}$ in the Xtra Power Mode at 2-mm distance [23]. In contrast to the irradiance of the Elipar FreeLight 2, the irradiance of the VALO light curing unit and without any ceramic disc initially increased for both curing modes. This may be explained by the fact that the VALO light curing unit is equipped with a lens which collimates the light beam. However, it is noteworthy that this initial increase in irradiance was extenuated when irradiance was measured through the ceramic discs. Both light curing units in all three modes showed a similar relative decrease in irradiance of more than $80 \%$ when measured through ceramic discs of $1.5-\mathrm{mm}$ thickness and of $95 \%$ or more when measured through ceramic discs of 3-mm thickness. At a given thickness, the leucite-reinforced glass ceramic IPS Empress CAD resulted in a somewhat smaller relative decrease in irradiance than the lithium-disilicate glass ceramic IPS e.max CAD regardless of the mode of the light curing units. This smaller decrease is related to a higher translucency of leucite-reinforced compared to lithium-disilicate glass ceramic materials, which has previously been described as caused by differences in microstructure with less dense crystals in the leucite-reinforced compared to the lithium-disilicate glass ceramic [24]. With a different second-generation LED curing unit (Bluephase 16i, Ivoclar Vivadent), Koch et al. reported decreases in irradiance of $60 \%$ through the leucite-reinforced glass ceramic ProCAD of 1-mm thickness and of more than $76 \%$ through 2 -mm discs. Through the lithium-disilicate glass ceramic Empress 2 of 1-mm thickness, the same authors reported higher decreases in irradiance of almost 90 and of $98 \%$ through the 2-mm discs [15], which resemble the decreases in irradiance of the present study. As regards spectra of the two light curing units, the Elipar FreeLight 2 light curing unit showed one distinct peak within a rather narrow spectrum, which is characteristic for secondgeneration LED curing units containing a single highpowered diode. The third-generation LED curing unit VALO contains four LEDs. One LED emits light with a wavelength of $405 \mathrm{~nm}$, leading to the distinct, smaller peak around $400 \mathrm{~nm}$. One LED emits light with a wavelength of $445 \mathrm{~nm}$, and two LEDs emit light with a wavelength of $465 \mathrm{~nm}$. The superimposition of these two peaks consequently led to the slightly stretched peak in the wavelength range of 440 to $470 \mathrm{~nm}$.

Measurement of DC showed significant differences between the resin cements as well as significant differences between the curing modes within one resin cement. Thus, the null hypothesis that all five resin cements would yield equal DC regardless of the curing mode was rejected. When self-cured only, four of the five resin cements showed significantly lower DC compared to the curing modes involving light curing. BeautiCem SA was the only resin cement which yielded an equal DC when self-cured as when light curing was involved. It must be mentioned, though, that
BeautiCem SA was not yet on the market when this study was initiated and thus, no literature data were available for comparison. In the self-curing mode, BeautiCem SA as well as SpeedCEM (which yielded a final DC of $56.4 \%$ ) reached a DC of around $5 \%$ after the first 2 to 4 min with a subsequent, gradual increase in DC. This suggests either the content of highly efficient self-curing initiators or the content of few inhibitors in these two resin cements. In group SC, the three other resin cements (i.e. Panavia F2.0, RelyX Unicem 2 Automix, and BisCem) reached a DC of around $5 \%$ only after the first 6 to $10 \mathrm{~min}$ with a rather slow subsequent increase in DC. In a previous study, DC of the conventional RelyX Unicem as well as BisCem was measured by micro ATR-FTIR for 10 min when self-cured and both RelyX Unicem as well as BisCem reached a DC of $11 \%$ [9]. For both resin cements, this DC is in a similar range as the DC measured in the present study: After the first 10 min, RelyX Unicem 2 Automix reached a DC of around $15 \%$, which then increased to the final DC of $24.7 \%$ after the measurement period of $20 \mathrm{~min}$. BisCem reached a DC of around $8 \%$ after the first $10 \mathrm{~min}$, which then increased to the final DC of $18.9 \%$ after $20 \mathrm{~min}$. In another study by Tezvergil-Mutluay et al., DC of the conventional RelyX Unicem as well as Panavia F2.0 was measured by ATR-FTIR for 15 min when self-cured, and RelyX Unicem reached a DC of $28.7 \%$ whereas Panavia F2.0 reached a DC of $27.9 \%$ [25], which is in accordance with the findings of the present study. Clinically and true to its category, Panavia F2.0 is used following application of a self-etch adhesive system (i.e. the ED Primer II). Thus, DC of an additional group of curing mode (group $\mathrm{SC}^{*}$ ) was measured for Panavia F2.0, mixed with ED Primer II. The accelerating effect of ED Primer has previously been described $[8,26]$, and the addition of ED Primer II led to a DC that was markedly higher than the $\mathrm{DC}$ of the curing modes involving light curing of Panavia F2.0. In group SC*, Panavia F2.0 together with ED Primer II, showed a DC of around $5 \%$ already after the first minute of self-curing.

For light curing of dental materials, there is a commonly assumed principle that similar radiant exposures lead to a similar total energy of light curing and thus to similar material properties. This principle is also known as the "total energy principle" or the "exposure reciprocity law" and is controversially discussed in literature. Whereas some studies have reported the exposure reciprocity law to be fulfilled (i.e. that similar radiant exposures with varying combinations of irradiance and light curing time lead to similar material properties) [18, 27], other studies have criticised the validity of the exposure reciprocity law. Different (filler/matrix) compositions of light curing dental materials may have a more pronounced influence on material properties than radiant exposure [17], and other authors have argued that 
irradiance and light curing time independently influence the curing process and consequently the material properties [19, 28, 29]. As regards light curing of the five resin cements in the present study, a higher irradiance generally did not result in significantly higher DC within a given resin cement. This may not be surprising because near identical radiant exposures were chosen and both irradiances and radiant exposures were very high, even with the Elipar FreeLight 2 in the Standard Mode. On the one hand, it might be of clinical interest to note that the light curing time was more than halved for the highest irradiance (i.e. $18 \mathrm{~s}$ for the VALO in the Xtra Power Mode) compared to the lowest irradiance (i.e. $40 \mathrm{~s}$ for the Elipar FreeLight 2 in the Standard Mode). On the other hand, both light curing times used for the VALO (i.e. the $32 \mathrm{~s}$ in the High Power Mode and the $18 \mathrm{~s}$ in the Xtra Power Mode) were longer than the actual light curing times stated by the manufacturer ( 8 to $12 \mathrm{~s}$ for the High Power Mode and 3 to $6 \mathrm{~s}$ for the Xtra Power Mode). Apart from this, however, direct light curing through a Mylar strip did not necessarily lead to significantly higher DC compared to groups where light curing had been performed through a ceramic disc. This mainly applied to Panavia F2.0, SpeedCEM, and BisCem. Within one of these three resin cements, no or only few significant differences between the curing modes were shown.

According to Ilie and Simon, these resin cements can be classified as resin cements with less sensitivity to the amount of light during light curing but with significant differences compared to self-curing alone. RelyX Unicem 2 Automix can be classified as a resin cement with higher sensitivity to the curing mode, with significant differences between self-curing and light curing as well as between the different groups involving light curing. BeautiCem SA can be classified as a resin cement with sensitivity to the amount of light during light curing but without differences between self-curing and light curing of lower irradiance (e.g. through the ceramic discs in the present study) [7]. This classification of the five resin cements was basically valid for both light curing units and corresponding modes. Unfortunately, limited manufacturers' information about the composition of resin cements hinders the interpretation of the above-mentioned classification. Finally, it must be noted that effects of post-curing of the resin cements (e.g. after $24 \mathrm{~h}$ or more) were not measured in the present study because of given technical specifications of the FTIR. Thus, a second study is currently in preparation in which the micromechanical properties (i.e. surface hardness as indirect determination of degree of conversion as well as elastic modulus) of the same resin cements and with the same curing modes are investigated after storage.
Acknowledgments S. Flury was supported by a grant (grant number: PB BEP3 136565) of the Swiss National Science Foundation (SNF, www.snf.ch). Furthermore, we thank S. Hayoz and Prof. Dr. J. Hüsler, Institute of Mathematical Statistics and Actuarial Science, University of Bern, for statistical analyses as well as Dr. A. Peutzfeldt for the scientific commentaries on the manuscript.

Conflict of interest The authors declare no conflicts of interest, real or perceived, financial or non-financial.

\section{References}

1. Fasbinder DJ, Dennison JB, Heys D, Neiva G (2010) A clinical evaluation of chairside lithium disilicate $\mathrm{CAD} / \mathrm{CAM}$ crowns: a two-year report. J Am Dent Assoc 141(Suppl 2):10S-14S

2. Frankenberger R, Taschner M, Garcia-Godoy F, Petschelt A, Krämer N (2008) Leucite-reinforced glass ceramic inlays and onlays after 12 years. J Adhes Dent 10:393-398

3. Fasbinder DJ (2006) Clinical performance of chairside CAD/CAM restorations. J Am Dent Assoc 137(Suppl):22S-31S

4. Radovic I, Monticelli F, Goracci C, Vulicevic ZR, Ferrari M (2008) Self-adhesive resin cements: a literature review. J Adhes Dent 10:251-258

5. van Dijken JW (2003) Resin-modified glass ionomer cement and self-cured resin composite luted ceramic inlays. A 5-year clinical evaluation. Dent Mater 19:670-674

6. van Dijken JW, Höglund-Aberg C, Olofsson AL (1998) Fired ceramic inlays: a 6-year follow up. J Dent 26:219-225

7. Ilie N, Simon A (2012) Effect of curing mode on the micromechanical properties of dual-cured self-adhesive resin cements. Clin Oral Investig 16:505-512

8. Flury S, Peutzfeldt A, Lussi A (2011) The effect of polymerization procedure on Vickers hardness of dual-curing resin cements. Am J Dent 24:226-232

9. Vrochari AD, Eliades G, Hellwig E, Wrbas KT (2009) Curing efficiency of four self-etching, self-adhesive resin cements. Dent Mater 25:1104-1108

10. Janda R, Roulet JF, Latta M, Kaminsky M, Rüttermann S (2007) Effect of exponential polymerization on color stability of resinbased filling materials. Dent Mater 23:696-704

11. Ferracane JL, Mitchem JC, Condon JR, Todd R (1997) Wear and marginal breakdown of composites with various degrees of cure. J Dent Res 76:1508-1516

12. Sideridou ID, Achilias DS (2005) Elution study of unreacted BisGMA, TEGDMA, UDMA, and Bis-EMA from light-cured dental resins and resin composites using HPLC. J Biomed Mater Res B Appl Biomater 74:617-626

13. Caughman WF, Caughman GB, Shiflett RA, Rueggeberg F, Schuster GS (1991) Correlation of cytotoxicity, filler loading and curing time of dental composites. Biomaterials 12:737-740

14. Kilinc E, Antonson SA, Hardigan PC, Kesercioglu A (2011) The effect of ceramic restoration shade and thickness on the polymerization of light- and dual-cure resin cements. Oper Dent 36:661-669

15. Koch A, Kroeger M, Hartung M, Manetsberger I, Hiller KA, Schmalz G, Friedl KH (2007) Influence of ceramic translucency on curing efficacy of different light curing units. J Adhes Dent 9:449-462

16. Jung H, Friedl KH, Hiller KA, Furch H, Bernhart S, Schmalz G (2006) Polymerization efficiency of different photocuring units through ceramic discs. Oper Dent 31:68-77

17. Hadis M, Leprince JG, Shortall AC, Devaux J, Leloup G, Palin WM (2011) High irradiance curing and anomalies of exposure reciprocity law in resin-based materials. J Dent 39:549-457 
18. Halvorson RH, Erickson RL, Davidson CL (2002) Energy dependent polymerization of resin-based composite. Dent Mater 18:463469

19. Musanje L, Darvell BW (2003) Polymerization of resin composite restorative materials: exposure reciprocity. Dent Mater 19:531-541

20. Nomoto R, Uchida K, Hirasawa T (1994) Effect of light intensity on polymerization of light-cured composite resins. Dent Mater J 13:198-205

21. Rueggeberg FA (2011) State-of-the-art: dental photocuring-a review. Dent Mater 27:39-52

22. Brunner E, Munzel U (2002) Nichtparametrische Datenanalyse (in German). Springer, Heidelberg

23. Price RB, Felix CM, Whalen JM (2010) Factors affecting the energy delivered to simulated class I and class $\mathrm{V}$ preparations. J Can Dent Assoc 76:a94
24. Ilie N, Hickel R (2008) Correlation between ceramics translucency and polymerization efficiency through ceramics. Dent Mater 24:908-914

25. Tezvergil-Mutluay A, Lassila LV, Vallittu PK (2007) Degree of conversion of dual-cure luting resins light-polymerized through various materials. Acta Odontol Scand 65:201-205

26. Faria-e-Silva AL, Moraes RR, Ogliari FA, Piva E, Martins LR (2009) Panavia F: the role of the primer. J Oral Sci 51:255-259

27. Emami N, Söderholm KJ (2003) How light irradiance and curing time affect monomer conversion in light-cured resin composites. Eur J Oral Sci 111:536-542

28. Asmussen E, Peutzfeldt A (2005) Polymerization contraction of resin composite vs. energy and power density of light-cure. Eur J Oral Sci 113:417-421

29. Peutzfeldt A, Asmussen E (2005) Resin composite properties and energy density of light cure. J Dent Res 84:659-662 